\title{
An IC architecture for RF Energy Harvesting systems
}

\author{
Leonardo Pantoli, Alfiero Leoni, Vincenzo Stornelli, and Giuseppe Ferri
}

\begin{abstract}
In this work we present an IC architecture for RF energy harvesting. The system has been designed with a $0.18 \mu \mathrm{m}$ CMOS SMIC technology and optimized at 900MHz. Simulation results have confirmed that the integrated system handles an incoming power typically ranging from $-25 \mathrm{dBm}$ to $20 \mathrm{dBm}$ by rectifying the variable input signals into a $\mathrm{DC}$ voltage source with an overall efficiency up to $50 \%$. The chip area estimation for the proposed system is as low as $3 \times 3 \mathrm{~mm}^{2}$.
\end{abstract}

Keywords-Energy harvesting; integrated circuits; autonomous devices; RF signals; low-power.

\section{INTRODUCTION}

In the last years, the number of RF sources that are available within urban environments is rapidly increasing thanks to the development of wireless communication systems that have replaced the massive cable interconnections. Beyond reducing the complexity of the systems, this tendency leads to the availability of many ambient energy still not used. Relying the electromagnetic (EM) energy, typically, a spectral survey reveals a wide range of energy contributions in the environment that are difficult to collect and store [1-4]. Till now, many RF energy harvesters have been presented in the literature, but only a few have been able to catch energy with good efficiency [5-10] being, especially at very low RF available energy, difficult to achieve good rectifiers. There are many problems that limit the collection of these resources, as for instance the not constant presence of the EM signals in the environment during the day and their spectral characteristics, due to modern communication codes, that spread the transmitted energy. From an electronic point of view, there are physical limitations that do not facilitate the realization of EM energy harvesters [5-12]. In particular, for what concerns the antenna and rectification section, its pattern and characteristics are strictly related to the target incoming frequency; another critical part is the regulation and storage section, because being not constant in time, the caught energy cannot be furnished to any devices but must be stored and provided on request, if available. Finally, any harvester systems may guaranty a conversion efficiency in order to provide more energy with respect to that it requires to properly work and be reasonably used. All these critical aspects are, in some way, mitigated by the recent advances and expansion that have involved the integrated technology.

Manuscript received January 15, 2017; revised May 11, 2017. Date of publication June 1, 2017.

Authors are with the Department of Industrial and Information Engineering and Economics, University of L'Aquila, 67100 Italy (e-mail: \{leonardo.pantoli, alfiero.leoni, vincenzo.stornelli, giuseppe.ferri\}@univaq.it).

Digital Object Identifier (DOI): 10.24138/jcomss.v13i2.377
The enhancements can be appreciated, at least, by two different points of view. Concerning the usefulness, in the last years both the size and power supply have been drastically decreased for many devices [13-15]. As a consequence, the idea to produce enough power to recharge the battery or directly supply the electronics is becoming even more consistent. About the reliability, the integrated circuit (IC) solutions will give more emphasis in the development of miniaturized energy harvesters with reduced power consumption and improved capability to track the energy variations of the incoming EM beam. These aspects will lead to energy harvesting systems with improved efficiency and advanced real-time operation capability. Also the receiving section may achieve further benefits from the IC technology with the possibility to realize integrated antenna of different topologies with compact dimensions and a wider operational bandwidth. The same authors have already developed and presented a discrete version of an energy harvester system capable to catch and manage RF radiation at $936 \mathrm{MHz}$ (used for mobile GSM transmissions) and $2.4 \mathrm{GHz}$ (frequency adopted for Wi-Fi internet connections) [16-18]. The aim of this work is to reproduce the promising results obtained with the discrete element board by means of an integrated technology: novel and completely integrable circuits and architectures are here presented for the first time. Each building block may be analyzed and reconsidered at technology level with important advantages in term of performance and miniaturization. Preliminary results are shown in this paper and have been obtained at simulation level using a $0.18 \mu \mathrm{m}$ CMOS SMIC technology with the aim to evaluate the expected additional benefits that can be achieved with future integration.

\section{Proposed Discrete ARChitecture}

A basic architecture for the realization of an energy harvester is shown in Fig. 1. Below the discrete, external, antenna, typically a matching network is used to transfer the incoming power towards the following signal managing circuits. In some cases, also a pass-band or a low-pass filter is used to eliminate unwanted frequency components that cannot be handled by the designed circuit. An important structure that must be carefully designed is the passive rectifier which must be able to perform the AC/DC transformation and it is usually designed with diode architectures. Finally, we usually have a storage section that implements the important function of energy storage or supply to external devices. 


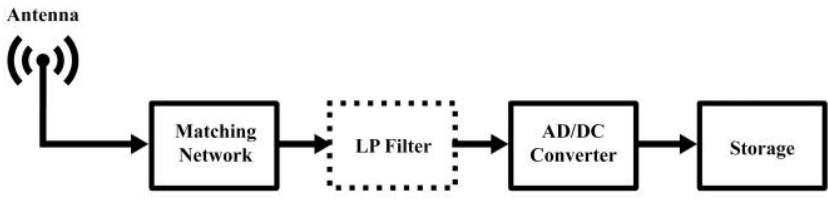

Fig. 1. Energy harvester simple architecture.

The harvester architecture that has been designed is illustrated in Fig. 2, while the detailed block scheme is shown in Fig. 3. It is conceived for operating in two separate bandwidth (the GSM sub-band of $936 \mathrm{MHz}$ and the WiFi channel at $2.4 \mathrm{GHz}$ ) and organized to handle different power levels on different channels in order to improve the conversion efficiency. There are two energy conversion channels ("Low Power" and "Medium Power" in Fig. 3), each of which optimized to work with a different range of incoming power levels at both the considered frequencies, being after the antenna considered a 1:100 transformer. The recovered energy is then manipulated by a common regulation and storage section. A feedback control network realized with a voltage comparator is responsible of switching the incoming power in the appropriate channel. It is an ultra-low power component that compares the rectified voltage with an internal voltage threshold and conveys the EM radiation towards the dedicated receiver. The "Low Power" way is conceived to manage an input power in the range $(-25: 5) \mathrm{dBm}$, while the "Medium Power" channel is optimized to work with input power levels going from $5 \mathrm{dBm}$ to $20 \mathrm{dBm}$. Both the channels foresee the AC/DC converter and different matching network, realized with passive components. In the discrete prototype, the following regulation and storage section have been realized with commercial components and makes use of an ultra-low voltage Step-Up Converter, the LT3108 from Linear Technology, that decouples the system from the generic load or device and boosts the incoming signal to the regulation section. Finally, a common load as a "storing section" is used for simulation and performance evaluation. In Fig. 3 for instance, a gas sensor has been used.

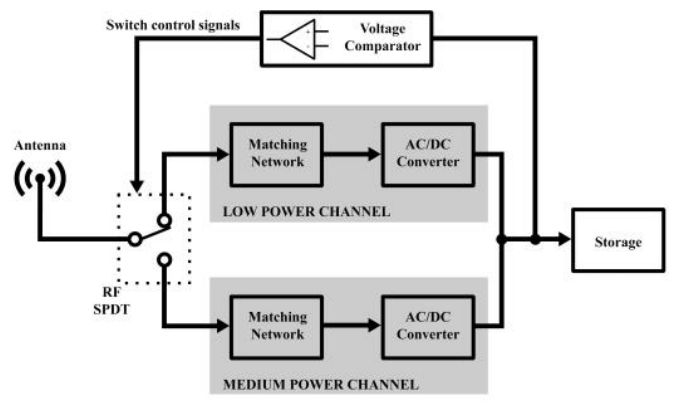

Fig. 2. Simplified scheme of the conceived architecture.

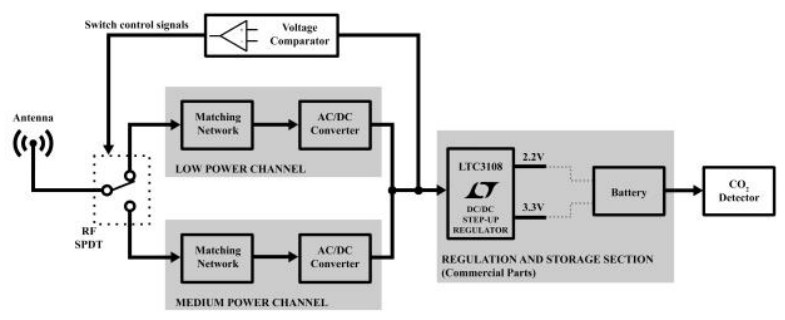

Fig. 3. Detailed block scheme of the designed, discrete, energy harvester architecture.

\section{INTEGRATED SOLUTION EVALUATION}

Concerning the novel IC solution, a feasibility study has been performed in order to obtain a reliable, miniaturized solution with improved performance both in term of conversion efficiency and harvested energy. The evaluation is performed at simulation level using a $0.18 \mu \mathrm{m}$ CMOS SMIC technology and considering a reference frequency of $900 \mathrm{MHz}$ that is used for both mobile phone service and digital television. In particular, the attention has been focused on the simplified architecture of Fig. 2, paying attention on the rectifiers, the voltage comparator and the DC/DC boost converter that are the most critical parts to catch and manage the incoming radiation (Fig. 4).

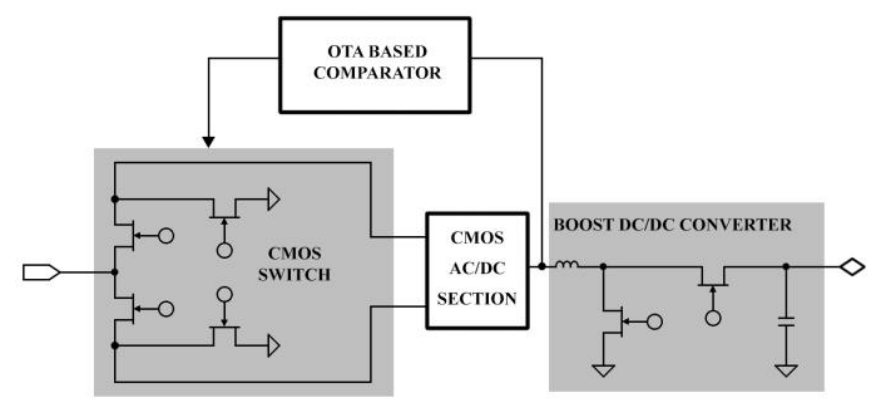

Fig. 4. Block scheme of the integrated version of the harvester.

About the antenna, a dipole antenna has been taken into account since it shows good performance on a wider bandwidth and can be successfully miniaturized adopting more complex geometry, as for instance in [19, 20]. In particular, a geometry folded dipole antenna similar to that proposed in [19] has been considered and is shown in Fig. 5. For system simulation purposes, the antenna has been simulated with Momentum software also considering a 1:100 $\mathrm{RF}$ transformer and represented by a Thevenin equivalent source considering its impedance.

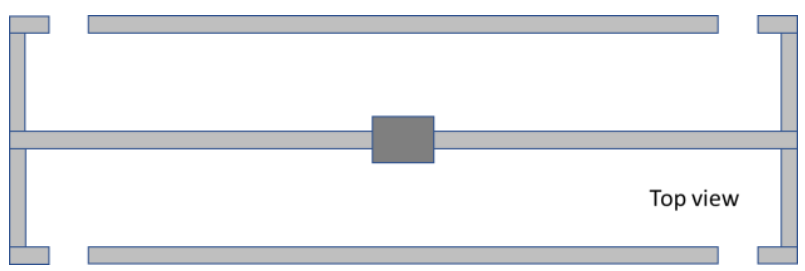

Fig. 5. Simplified representation of the considered folded dipole antenna.

The RF Switch can be easily realized with parallel MOS transistors controlled by means of gate voltages, as shown in the miniature of Fig. 4. The comparator is a crucial element in the RF chain since even if it makes possible to double the power management chains, it requires a bias voltage and a reference voltage to set a suitable voltage threshold. The proposed solution concerns the realization of the comparator with a couple of low-voltage, low-power operational transconductance amplifiers (OTAs) [21,22] that allow to minimize the requested power to drive the switch. The circuit here adopted for the OTA is shown in Fig. 6 and it requires a very low biasing level $(<1 \mu \mathrm{W})$ that can be easily recovered 
from the storage section itself. Obviously, we are supposing the system will be provided of a rechargeable battery pack, initially charged, so avoiding the use of an external supply voltage for a while, until the incoming RF radiation arises to a sufficient value to auto-sustain the circuit functionality. In detail, the OTA has two stages: an input symmetrical OTA $(M 1-M 9)$ that decreases systematic and random offsets; a source degenerated output stage $(M 10-M 13)$ that allows to obtain a full dynamic output range. Compensation is ensured by $R C$ series network, while a high slew-rate value is obtained properly dimensioning a suitable $V_{t h}$-dependent current generator through (M14-M17, $R I)$. This current reference uses a "start-up" circuit.

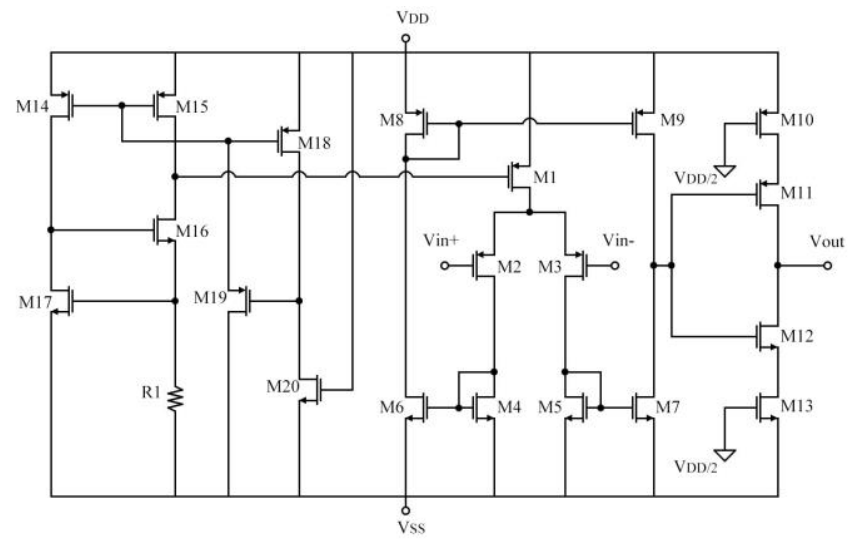

Fig. 6. Designed LV-LP OTA used for the comparator.

Concerning the voltage rectifier, being the two channels of the harvester dimensioned to handle quite different input power levels, it has been necessary to develop different solutions for this building block in order to have at the same time good sensitivity and the capability to manage large input power levels, so ensuring a wide dynamic range. The solution adopted for the medium-power channel is reported in Fig. 7 and derived by the architecture proposed in [22]. It is a full-wave gate cross-coupled rectifiers where the transistors in the two main branches are cross-coupled. This configuration limits the associated voltage drop due to the voltage threshold of the MOS transistor and allows to obtain a higher power efficiency towards classical diode-based solutions. The low-power channel has been conceived with a different scheme, keeping advantages of the solution always proposed in [23]. Since this channel is devoted to handle low-power levels, improved performance in term of efficiency and sensitivity are necessary. The adopted solution implements two MOS switches with very low effective threshold voltage to replace the classical diodeconnected pMOS transistors usually employed. In addition, this architecture also take advantages from the cross coupled structures that are applied to the MOS transistors and this allows to drive the transistors with a larger voltage swing, increasing the output voltage and improving the power conversion efficiency. A dynamic bulk biasing circuit for diode-connected transistors $M 5$ - M6 is also used for reducing the circuit dropout voltage and power dissipation at start up.

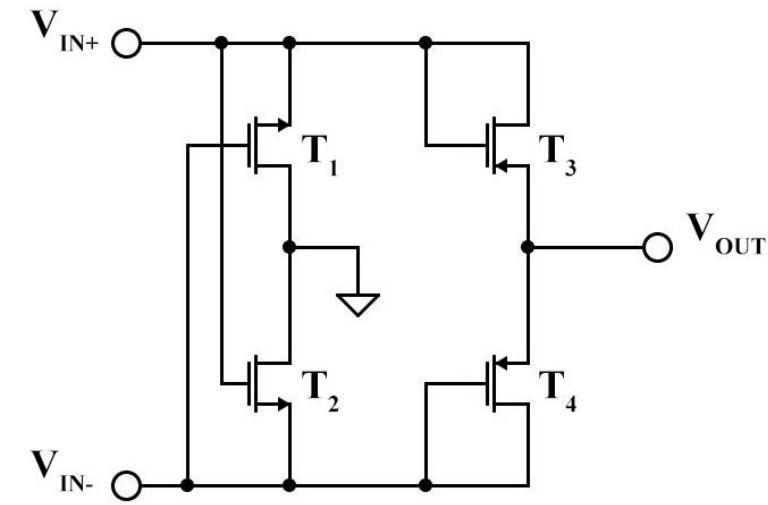

Fig. 7. Schematic of full-wave medium-power channel rectifier as proposed in [23].

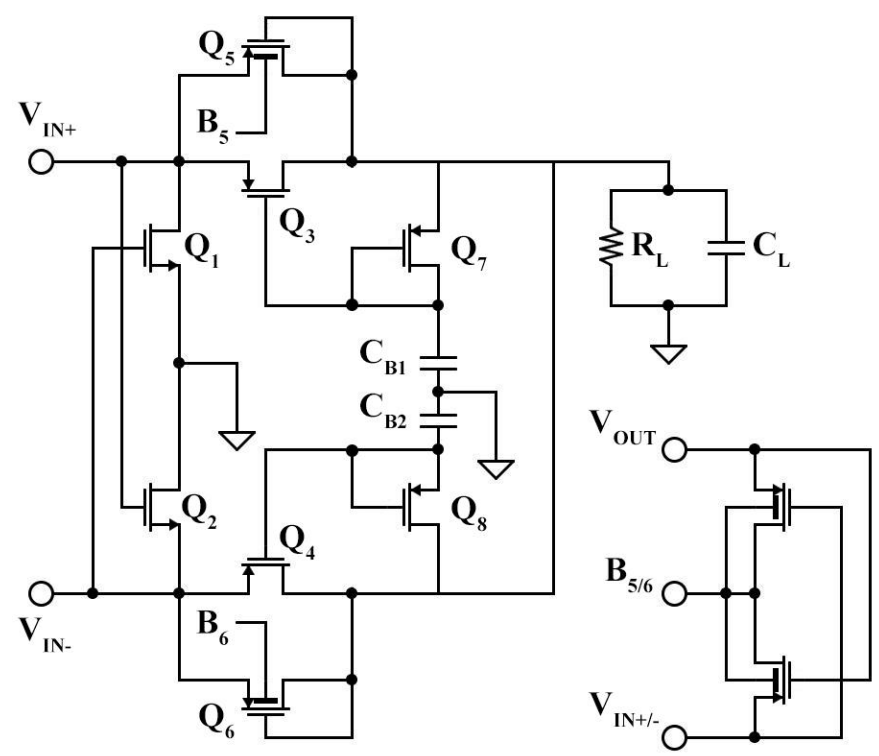

Fig. 8. Schematic of full-wave low-power channel rectifier as proposed in [23].

Finally, the solution illustrated in Fig. 9 has been adopted for the Boost DC/DC converter [24]. In this design, two instances of the low-power OTA (that is shown in Fig.6) are used in a charge pump configuration to multiply the input voltage. The first OTA acts as a relaxation oscillator, serving as the master charge-pump clock, while the second amplifier is configured as a comparator slaved to the timing cycle established by the first one. Considering the same definition of the Efficiency parameter as in [18], that is, the ratio between the incoming power and the rectified power, preliminary simulation results have confirmed that the integrated system handles an incoming power typically ranging from $-25 \mathrm{dBm}$ to $5 \mathrm{dBm}$ by rectifying the variable input signals into a $\mathrm{DC}$ voltage source with an overall efficiency up to 50\%. In Fig. 10 and Fig. 11, simulation results concerning the conversion efficiency on the two channels are shown confirming the suitability of the proposed circuits towards portable system applications. The chip area estimation for the proposed system is as low as $3 \times 3 \mathrm{~mm}^{2}$. 


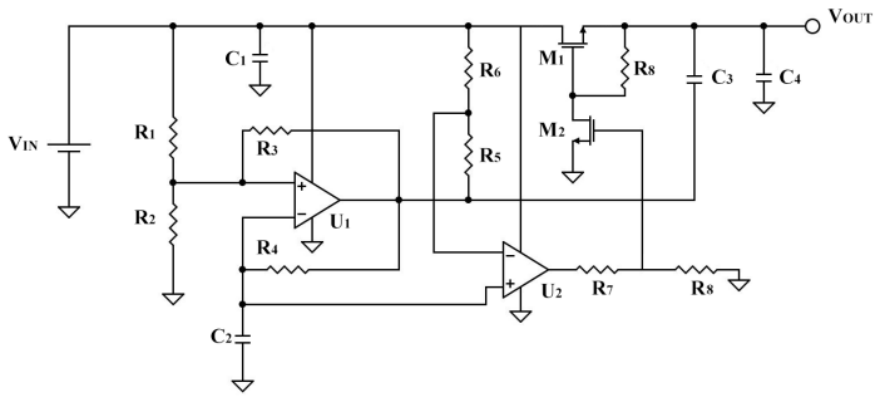

Fig. 9. Simplified schematic of the boost DC/DC converter.

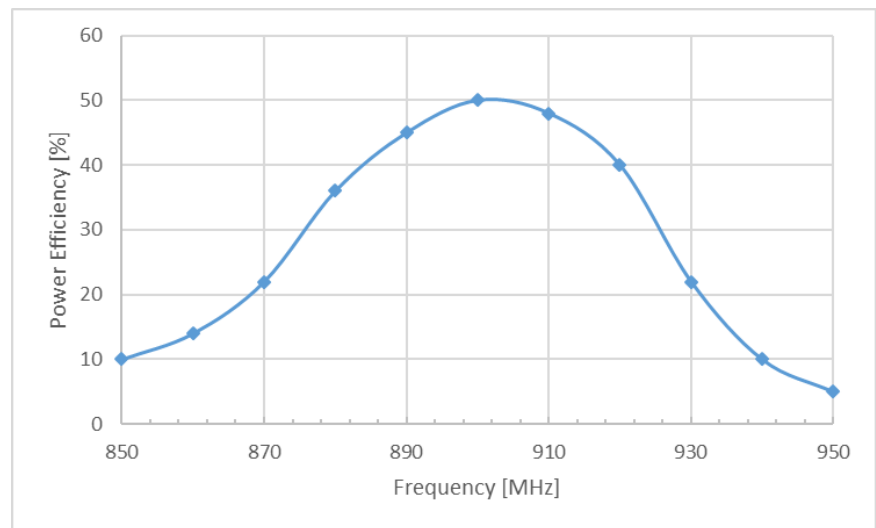

Fig. 10. Conversion efficiency for the low power channel at $0 \mathrm{dBm}$ input power

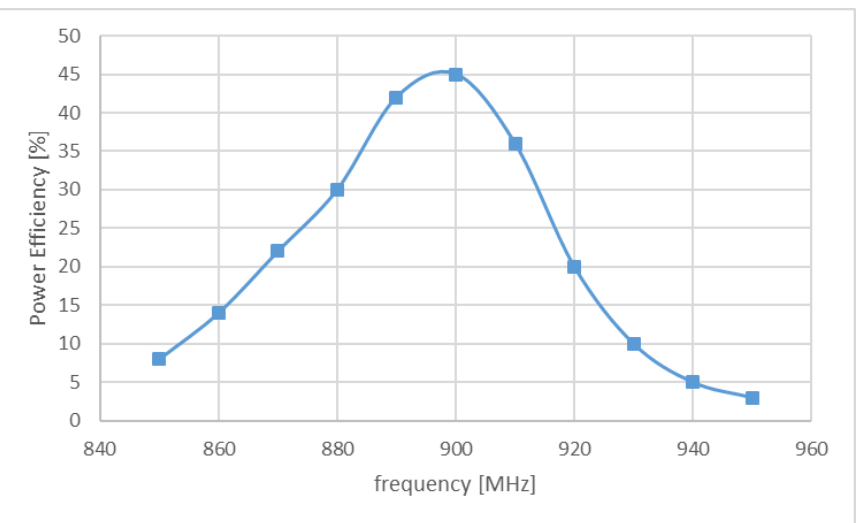

Fig. 11. Conversion efficiency for the medium power channel at 10 $\mathrm{dBm}$ input power

\section{CONCLUSION}

We have presented preliminary simulation results about IC architecture for the realization of a dual band RF energy harvesting system. The solution here addressed takes advantages of the results already obtained by the same authors with a discrete prototype board operating at both GSM and Wifi frequencies. The integrated version has utilized models taken from a $0.18 \mu \mathrm{m}$ CMOS SMIC technology and the system has been dimensioned at $900 \mathrm{MHz}$. Circuitry details of the main building block have been described and preliminary results demonstrate the feasibility of the proposed solution and good reliability in terms of its performances.

\section{REFERENCES}

[1] K. Al Agha, M.-H. Bertin, T. Dang, A. Guitton, P. Minet, T. Val, and J.-B. Viollet, Which Wireless Technology for Industrial Wireless Sensor Networks? The Development of OCARI Technology, IEEE Transactions on Industrial Electronics, vol.56, no.10, 2009, pp. 4266-4278.

[2] V. C. Gungor and G. P. Hancke, Industrial Wireless Sensor Networks: Challenges, Design Principles, and Technical Approaches, IEEE Transactions on Industrial Electronics, vol.56, no.10, 2009, pp. 4258-4265.

[3] Y. Levron and D. Shmilovitz, A Power Management Strategy for Minimization of Energy Storage Reservoirs in Wireless Systems With Energy Harvesting, IEEE Transactions on Circuits and Systems I: Regular Papers, (2010) pp. 1-11.

[4] H. Xiao, H. Shao, K. Yang, F. Yang, W. Wang, Multiple Timescale Energy Scheduling for Wireless Communication with Energy Harvesting Devices, Radioengineering Journal, vol. 21 (2012), Is. 3.

[5] E. Khansalee, Yan Zhao, E. Leelarasmee, K.Nuanyai, A dualband rectifier for RF energy harvesting systems, 11th International Conference on Electrical Engineering/Electronics, Computer, Telecommunications and information Technology ECTI-CON (14-17 May 2014), pp. 1-4.

[6] M. Gutierrez, A. Shahidi, D. Berdy, D. Peroulis, Design and characterization of a low frequency 2-dimensional magnetic levitation kinetic energy harvester, ELSEVIER Sensors and Actuators A: Physical, vol. 236 (2015), pp. 1-10.

[7] M. Piñuela, P. D. Mitcheson and S. Lucyszyn, "Ambient RF Energy Harvesting in Urban and Semi-Urban Environments," in IEEE Transactions on Microwave Theory and Techniques, vol. 61, no. 7, pp. 2715-2726, July 2013.

[8] M. Sun, D. Ranasinghe and S. F. Al-Sarawi, "RF energy harvester with peak power conversion efficiency tracking," 2016 IEEE Asia Pacific Conference on Circuits and Systems (APCCAS), Jeju, South Korea, 2016, pp. 107-110.

[9] P. D. Gleonec, J. Ardouin, M. Gautier and O. Berder, "Architecture exploration of multi-source energy harvester for IoT nodes," 2016 IEEE Online Conference on Green Communications (OnlineGreenComm), Piscataway, NJ, USA, 2016, pp. 27-32.

[10] J. J. Shi and X. D. Huang, "Electrostatic energy harvester based on charge-trapping nonvolatile memory structure," 2016 IEEE International Conference on Electron Devices and Solid-State Circuits (EDSSC), Hong Kong, Hong Kong, 2016, pp. 153-156.

[11] Zhongjun Wang, Wanrong Zhang, Dongyue Jin, Hongyun Xie and Xiaoqiang Lv, "A full-wave RF energy harvester based on new configurable diode connected MOSFETs," 2016 IEEE International Conference on Microwave and Millimeter Wave Technology (ICMMT), Beijing, 2016, pp. 117-119.

[12] Panagiotis Broutas, Harry Contopanagos, Dimitrios Tsoukalas, Stavros Chatzandroulis, A RF power harvester with integrated antenna capable of operating near ground planes, Sensors and Actuators A: Physical, Volume 186, October 2012, Pages 284288, ISSN 0924-4247.

[13] L. Pantoli, V. Stornelli and G. Leuzzi, "Class AB tunable active inductor," in Electronics Letters, vol. 51, no. 1, pp. 65-67, 18 2015.

[14] L. Pantoli, V. Stornelli, G. Leuzzi, "Tunable active filters for RF and microwave applications", J. Circuits Syst. Comput., 23 (2014).

[15] L. Pantoli, V. Stornelli and G. Leuzzi, "Low-noise tunable filter design by means of active components", Electronics Letters, vol. 52 , no. 1, pp. 86-88, Jan. 2016.

[16] L. Pantoli, A. Leoni, V. Stornelli and G. Ferri, "Energy harvester for remote sensors systems," 2016 International Multidisciplinary Conference on Computer and Energy Science (SpliTech), Split, 2016, pp. 1-3. 
[17] P. Di Marco, A. Leoni, L. Pantoli, V. Stornelli and G. Ferri, "Remote sensor networks with efficient energy harvesting architecture," 2016 12th Conference on Ph.D. Research in Microelectronics and Electronics (PRIME), Lisbon, 2016, pp. 14.

[18] P. Di Marco, V. Stornelli, G. Ferri, L. Pantoli, A. Leoni, Dual band harvester architecture for autonomous remote sensors, Sensors and Actuators A: Physical, Volume 247, 15 August 2016, Pages 598-603, ISSN 0924-4247.

[19] Tashi, M. S. Hasan and H. Yu, "Design and simulation of UHF RFID tag antennas and performance evaluation in presence of a metallic surface," 2011 5th International Conference on Software, Knowledge Information, Industrial Management and Applications (SKIMA) Proceeding $s$, Benevento, 2011, pp. 1-5.

[20] L. Pantoli, V. Stornelli, G. Leuzzi and A. Di Carlofelice, "A single transistor post selector active tunable filter for radio receivers applications," 2014 International Workshop on Integrated Nonlinear Microwave and Millimetre-wave Circuits (INMMiC), Leuven, 2014, pp. 1-3.

[21] G. Ferri, V. Stornelli, A. Celeste, "Integrated rail-to-rail lowvoltage low-power enhanced DC-gain fully differential operational transconductance amplifier", ETRI Journal, Volume 29, Issue 6 Pages 785-792, 2007.

[22] A. De Marcellis, A. Depari, G. Ferri, A. Flammini, D. Marioli, V. Stornelli, A. Taroni, "A CMOS integrable oscillator-based front-end for high dynamic range resistive sensors", IEEE Transactions on Instrumentation and Measurement, Vol. 57, Issue 8, Pages: 1596 - 1604, 2008.

[23] S. S. Hashemi, M. Sawan and Y. Savaria, "A High-Efficiency Low-Voltage CMOS Rectifier for Harvesting Energy in Implantable Devices," in IEEE Transactions on Biomedical Circuits and Systems, vol. 6, no. 4, pp. 326-335, 2012.

[24] "A Microwatt Charge Pump Boosts $1 \mathrm{~V}$ to $1.8 \mathrm{~V}$ at $90 \%$ Efficiency, Providing "Always On" Standby Power for Microcontrollers", Silicon Lab Technical paper.

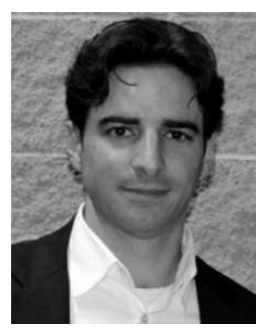

Leonardo Pantoli Leonardo Pantoli received the Laurea (M.S.) degree in electronic engineering (cum laude) and Ph.D. degree in electrical and information engineering from the University of L'Aquila, L'Aquila, Italy, in 2006 and 2010, respectively. In 2007 and 2008, he spent several months with the Communications Engineering Department, University of Cantabria, Santander, Spain, and the C2S2 Department, XLIM Research Institute, Brive La Gaillarde, France. He is currently a Researcher with the University of L'Aquila. His research activities include the development of methods and algorithms for the design of microwave nonlinear circuits, stability analysis under the large-signal regime, active filters, and monolithic microwave integrated circuit (MMIC) design.

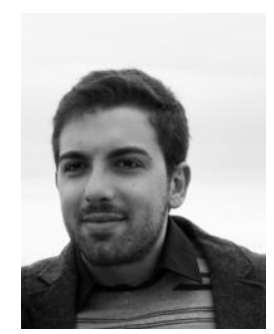

Alfiero Leoni was born in L'Aquila, Italy. He received the Bachelor "Laurea" degree in 2013 and master "Laurea" degree (cum laude) in electronic engineering in 2016. Several months before his master laurea degree, he joined as an external collaborator the Department of Industrial and Electronic Engineering of the University of L'Aquila where he is actually involved as Ph.D. student. His research activity mainly consists in the design of analog electronic circuits and systems for energy harvesting, industrial and microwave applications.

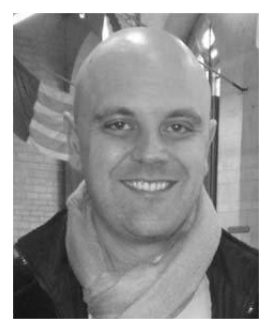

Vincenzo Stornelli was born in Avezzano, Italy. He received the "Laurea" degree (cum laude) in electronic engineering in 2004. In October 2004, he joined the Department of Electronic Engineering, University of L'Aquila, L'Aquila, Italy, where he is involved as Associate Professor with problems concerning current mode applications; physicsbased simulation; computer-aided design modeling characterization and design analysis of active microwave components, circuits, and subsystems; and the design of integrated circuits for RF and sensor applications. He serves as a reviewer for several international journals. His research interests include several topics in computational electromagnetics, including microwave antenna analysis for outdoor ultrawideband applications.

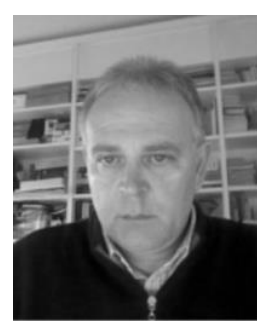

Giuseppe Ferri was born in L'Aquila, Italy. He received the "Laurea" degree (cum laude) in electronic engineering in 1988. In 1991, he joined the Department of Electronic Engineering, University of L'Aquila, L'Aquila, Italy, where he is actually a full professor of Electronics and Microelectronics at the University of L'Aquila, Italy. His research activity mainly concerns the design of analog electronic circuits for integrated sensor applications both in voltage and in current-mode. In this field of research he is author or coauthor of 2 patents, 2 international books, one book chapter and more than 330 publications in international journals and conference proceedings. He is an IEEE senior member and Editor of Sensors and of Journal of Circuits, Computers and Systems. 